\title{
Étude du comportement d'un échangeur à plaques Vicarb utilisé pour la pasteurisation du lait
}

\author{
par \\ M. LALANDE, G. CORRIEU, J. P. TISSIER \\ et R. FERRET \\ I.N.R.A., Laboratoire de Génie Industriel \\ 369, rue Jules-Guesde - 59650 Villeneuve-d'Ascq
}

R é s u m é

Les auteurs décrivent le comportement mécanique, hydrodynamique et thermique d'un échangeur de chaleur à plaques (Vicarb, modèle V7) adapté à la pasteurisation du lait. Tout en concluant au parfait fonctionnement de l'échangeur, et à ses possibilités d'utilisation dans l'ensemble des industries agro-alimentaires, ils mettent en évidence les limites des connaissances théoriques actuelles.

\section{S u m m a r y}

The mechanical, hydrodynamical and thermal behaviour of the heat-plate exchanger Vicarb V7 used in milk pasteurization is described. Our studies have shown the good performance of this equipment and its potential adaptation to other food processes. Yet the authors stress the present limits of theoretical knowledge in this field.

\section{INTRODUCTION}

Depuis plusieurs années, les échangeurs de chaleur à plaques prennent une place sans cesse croissante dans l'équipement des industries agro-alimentaires, et de l'industrie laitière en particulier. Cette évolution apparaît pour l'instant irréversible, en raison des nombreux avantages qu'ils présentent par rapport aux échangeurs tubulaires. Ils conduisent, en effet, à d'excellents rapports surface d'échange/volume 
qui limitent leur encombrement et simplifient leur intégration aux unités de production. Il est facile, en modifiant leur configuration générale, de les adapter à un programme thermique différent de celui initialement prévu; par exemple, l'augmentation du coût de l'énergie a entraîné celle des quantités de chaleurs récupérées. Les échangeurs à plaques sont aptes au nettoyage en place, ce qui limite les frais d'entretien. Vinconneau et Crevel [1], analysant dans le cadre d'une application à l'industrie sucrière les coûts d'investissement et de fonctionnement d'un modèle tubulaire et à plaques, concluent à la supériorité de ce dernier.

Trois causes principales, liées à la conception des échangeurs à plaques, expliquent qu'ils fournissent de meilleurs coefficients généraux d'échange que les échangeurs tubulaires et, par conséquent, qu'ils exigent pour un programme thermique donné, des surfaces d'échange moindres. Il s'agit :

$1^{\circ}$ De la réduction du diamètre hydraulique nominal, dont les valeurs, pour les échangeurs à plaques, se situent entre 5 et $10 \mathrm{~mm}$ alors que des tubes de diamètre inférieur à $20 \mathrm{~mm}$ ( $3 / 4$ de pouce) ne sont jamais utilisés.

$2^{\circ}$ De la diminution de l'épaisseur de la paroi d'échange. Les techniques d'emboutissage actuelles permettent d'obtenir des plaques d'épaisseur comprise entre 0,6 et $0,8 \mathrm{~mm}$ contre 1 à $1,5 \mathrm{~mm}$ pour les tubes.

$3^{\circ}$ De l'augmentation de turbulence, à débit constant, provoquée par la structure ondulée des plaques qui conduit à des coefficients de film améliorés.

Il est surprenant de constater, à la lecture d'ouvrages spécialisés $[2,3,4]$, qu'aucun travail théorique complet établissant les relations entre les modèles d'écoulement (turbulence, perte de charge, couche limite) et le profil des plaques n'ait été réalisé, sauf peut-être par les constructeurs. Ceux-ci utilisent néanmoins pour calculer leurs échangeurs, les formules traditionnelles, conçues et vérifiées pour les conduits tubulaires, après identification expérimentale des coefficients afin qu'ils s'appliquent aux plaques.

Les industriels souhaitant s'équiper d'échangeurs à plaques, se voient proposer différents matériels d'origine étrangère parmi lesquels le choix n'est pas facile. Les critères de comparaison restent plus ou moins flous (il est ridicule d'employer par exemple, le coefficient général d'échange sans préciser le programme thermique auquel il correspond, en particulier les débits de circulation des fluides) et à la discrétion des constructeurs. Par ailleurs, les différents paramètres intervenant dans le calcul des échangeurs, mal connus des utilisateurs, conditionnent les surfaces d'échanges proposées, et par suite certaines performances futures telles la durée de fonctionnement entre deux nettoyages. En conséquence, le prix n'est pas toujours un critère de comparaison significatif. 
A notre connaissance, seules les performances du modèle «Varitherm 40 » construit par Ahlborn ont fait l'objet d'une publication [5]. Nous présentons pour notre part, les conclusions de diffénents essais de pasteurisation du lait réalisés sur un échangeur, modèle V7, de la société Vicarb, seul constructeur français d'échangeurs à plaques.

Nous avons choisi la pasteurisation du lait pour deux raisons :

- les propriétés encrassantes du lait posent un problème technique difficile à résoudre ;

- l'industrie laitière occupe, sur le plan économique, en particulier au niveau des investissements, la première place du secteur agro-alimentaire [6].

\section{DESCRIPTION DU MATERIEL.}

Le pasteurisateur, représenté schématiquement figure 1, comprend quatre "zones thermiques":

- la zone de récupération de chaleur (notée B) dans laquelle le lait froid entrant est réchauffé par le lait pasteurisé en cours de refroidissement ;

- la zone de chauffage (notée C) dans laquelle le lait préchauffé est porté à sa température de pasteurisation au moyen d'énergie calorifique fournie par un courant d'eau chaude ;

- la zone de chambrage (notée $\mathrm{D}$ ) où le lait est maintenu à sa température de pasteurisation pendant une durée fixée par le rapport entre le volume du chambreur et le débit de lait ;

- la zone de réfrigération (notée $\mathrm{A}$ ) où le lait prérefroidi est amené à sa température de stockage ( $4^{\circ} \mathrm{C}$ environ) au moyen d'énergie frigorifique fournie par un courant d'eau glacée.

Il est constitué de plaques de $0,075 \mathrm{~m}^{2}$ de surface utile (modèle V7). Sa configuration a été établie au moyen du programme informatique de calcul habituellement utilisé par Vicarb, à partir du programme thermique ci-dessous :

- débit de lait (grand mélange, cru, entier) : $300 \mathrm{l} / \mathrm{h}$;

- température d'entrée du lait : $5^{\circ} \mathrm{C}$;

- température de pasteurisation : $85^{\circ} \mathrm{C}$;

- température du lait préchauffé : $69^{\circ} \mathrm{C}$;

- température du lait prérefroidi : $21^{\circ} \mathrm{C}$;

- température de sortie du lait : $5^{\circ} \mathrm{C}$;

- temps de chambrage : $12 \mathrm{~s}$ à $85^{\circ} \mathrm{C}$;

— débit d'eau glycolée : $9001 / \mathrm{h}$;

- température d'entrée de $1^{\prime}$ eau glycolée : $-5^{\circ} \mathrm{C}$; 


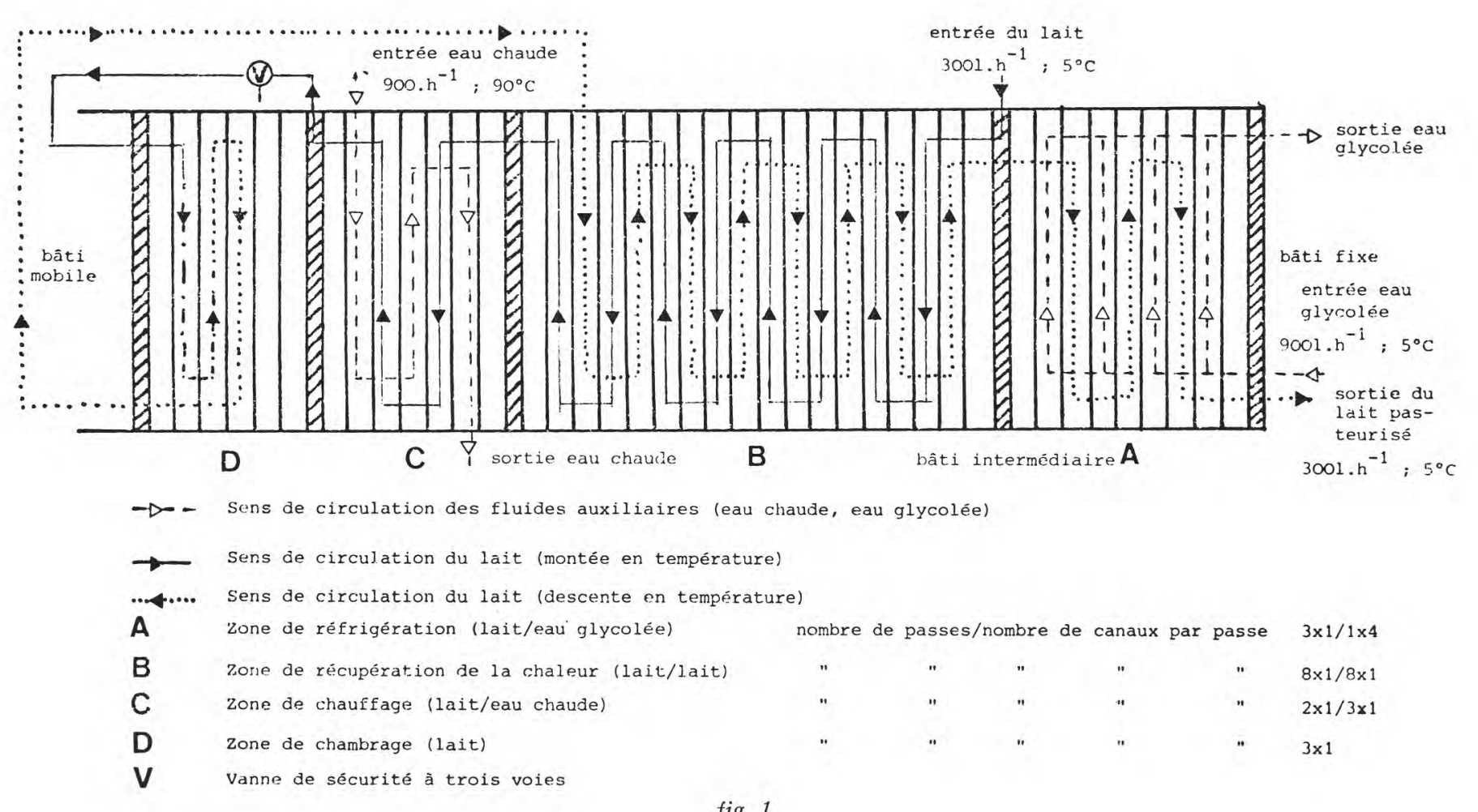

fig. 1

Représentation schématique du pasteurisateur (configuration et programme thermique) 
- débit d'eau chaude : $9001 / \mathrm{h}$;

- température d'entrée de l'eau chaude : $90^{\circ} \mathrm{C}$;

- coefficient d'encrassement (côté lait) : $1.10^{-4} / \mathrm{h} / \mathrm{m}^{2} /{ }^{\circ} \mathrm{C} / \mathrm{Kcal}$;

- coefficient d'encrassement (côté eau) : $2.10^{-5} / \mathrm{h} / \mathrm{m}^{2} /{ }^{\circ} \mathrm{C} / \mathrm{Kcal}$;

- conductibilité thermique de la paroi (plaques en acier inoxydable AISI $316 \mathrm{TI}) 14 \mathrm{Kcal} / \mathrm{h} / \mathrm{m}^{2} /{ }^{\circ} \mathrm{C}$;

- pourcentage d'énergie récupérée : 80 p. 100.

La température de pasteurisation est maintenue constante au moyen d'une boucle de régulation automatique dont l'actionneur agit sur le débit d'eau chaude circulant dans le tronçon de chauffage. Nous avons choisi ce fluide car il est sur le plan énergétique, plus économique que la vapeur et devrait prendre une importance croissante dans les unités de pasteurisation industrielles.

Nous avons limité à 80 p. 100 l'énergie récupérée dans la zone B (préchauffage du lait) afin que cette dernière ne constitue pas l'essentiel de l'échangeur au détriment de la zone de chauffage du lait (zone C). La récupération de 90 à 95 p. 100 de l'énergie (pourcentage courant dans l'industrie laitière) nous aurait conduit dans les conditions choisies à une zone $\mathrm{C}$ de très petite taille, peu significative pour l'extrapolation des résultats.

Les essais ont été réalisés en intégrant le pasteurisateur à une plate-forme semi-industrielle complètement équipée (bacs de stockage du lait, installation de nettoyage en place, réserves d'eau chaude et d'eau glacée, etc.). Un ensemble de capteurs performants (température, débit, pression) fournit les mesures indispensables à leur exploitation. Un minicalculateur (le Picolog 80 de Leanord) assurant la conduite automatique de l'ensemble de la plate-forme, centralise et traite ces données.

\section{CONDUITE DES ESSAIS - RESULTATS}

Le comportement d'un échangeur de chaleur s'apprécie au moyen de plusieurs critères. Les aspects mécaniques, hydrodynamiques et thermiques caractérisent l'échangeur en tant que tel. Son domaine d'application nous a conduit à considérer également l'encrassement des plaques, leur nettoyage et la qualité bactériologique du lait traité.

Nous nous attachons à décrire les observations et résultats correspondant à chacun de ces aspects.

\section{Tenue des joints}

Soumis à des contraintes mécaniques, thermiques et chimiques souvent sévères, les joints constituent le point faible des échangeurs à plaques. 
Dans le cas présent, la tenue mécanique des joints s'est révélée particulièrement satisfaisante. Les protocoles expérimentaux nous conduisent à démonter deux fois le pasteurisateur au cours d'un essai. Environ cinquante opérations de démontage-remontage (espacées sur 8 mois) n'ont provoqué aucune altération des joints. Le pasteurisateur ne présente aucune fuite, aussi bien à chaud qu'à froid. Cette propriété, qui n'existe pas chez certains modèles concurrents, s'explique par la largeur de portée des joints, la qualité du collage et la rigidité de l'ensemble. Assemblées, les plaques comportent de nombreux points de contact qui confèrent aux échangeurs Vicarb la particularité d'accepter des pressions de travail de l'ordre de 12 bars.

La tenue thermique ( $100^{\circ} \mathrm{C}$ environ côté eau chaude) et chimique (lavages acides et alcalins) des joints est aussi satisfaisante.

\section{Résistance à la corrosion}

Les corrosions localisées (caverneuse, par piqûres) sont assez fréquentes sur de nombreux matériels de l'industrie agro-alimentaire et du secteur laitier en particulier. Les produits de nettoyage et de désinfection utilisés pour assurer la propreté des installations et les saumures de refroidissement sont généralement à l'origine de l'apparition et du développement de telles corrosions $[7,8]$.

Nous avons choisi d'utiliser des plaques en acier inoxydable du type Z8 CNDT 17-12 (AISI $316 \mathrm{Ti}$ ) dont la composition classique est : 0,08 p. 100 de carbone, 17 p. 100 de chrome, 12 p. 100 de nickel, 2,5 p. 100 de molybdène et 0,32 p. 100 de titane. Parmi les nuances d'aciers inoxydables courantes au chrome-nickel, c'est celle qui présente la meilleure résistance à la corrosion.

Les résultats ont confirmé cette analyse puisque 18 mois d'essais, comportant en particulier la désinfection systématique du pasteurisateur au moyen d'une solution d'hypochlorite de sodium, à température ambiante, n'ont pas permis de relever la moindre corrosion.

\section{Dispersion des temps de séjour}

Si l'écoulement du lait au sein du pasteurisateur était de type "piston ", c'est-à-dire sans mélange, le temps de séjour de tous les germes contenus dans le lait serait égal et leur destruction thermique homogène. Mais comme tout appareil, le pasteurisateur a un effet dispersant qui influence la destruction des germes. Rao et Loncin [9] ont présenté une étude théorique de l'effet cumulé de ces deux phénomènes. Ils expriment, en particulier, le temps nécessaire à la destruction d'une certaine quantité de germes en fonction de la fonction de répartition des temps de séjour. Par ailleurs, Thor et Loncin [10] ont montré que les opérations de rinçage pouvaient être décrites par des cinétiques simples, partiellement basées sur la notion de temps de séjour moyen. 
Pour analyser la dispersion des temps de séjour causée par l'échangeur, nous avons choisi de suivre l'évolution d'un front eausolution de soude à $20 \mathrm{~g}$ par litre au moyen de mesures de résistivité effectuées sur des échantillons significatifs de la solution sortant de l'échangeur.

Quatre débits compris entre 200 et 5401 par heure ont été étudiés lors du remplacement de l'eau contenue dans l'échangeur par de la soude et lors de l'opération inverse. Les résultats expérimentaux sont rapportés à la figure 2 .
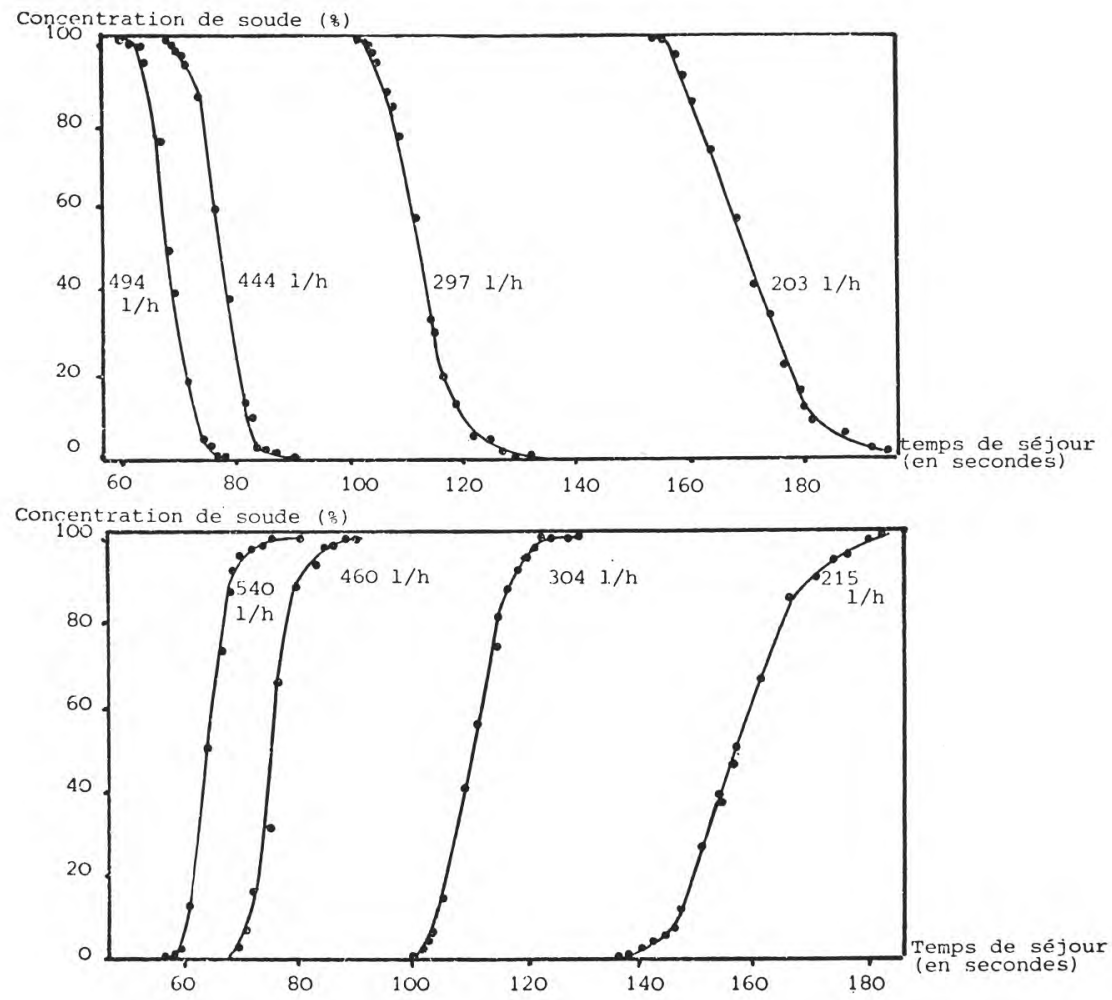

Remplacement de l'eau par la solution de soude à 28

fig. 2

Evolution de la concentration de soude à la sortie du pasteurisateur en fonction du temps pour différents débits. 
Leur examen visuel montre que le front présente une allure semblable quel que soit le remplacement effectué. La dispersion est d'autant plus faible que le débit de circulation est important.

L'analyse mathématique des résultats expérimentaux permet de montrer que le phénomène de dispersion peut être décrit (pour des pourcentages de soude compris entre 6 et 94 p. 100) soit par une loi normale, soit par le modèle des mélangeurs parfaits en série $[9,11]$. Dans ce dernier cas, l'identification du modèle montre que l'échangeur étudié correspond à environ 400 de ces mélangeurs, et est donc relativement peu dispersant.

Ces travaux, généralisés à la gestion des opérations de rinçage des unités de pasteurisation, feront l'objet de publications spécifiques.

\section{Pertes de charge}

La connaissance des pertes de charge des différentes parties d'un échangeur est nécessaire pour le calcul des pompes assurant la circulation des fluides.

Au moyen d'un capteur différentiel de pression et d'un débitmètre électromagnétique, nous avons étudié l'évolution de la perte de charge du circuit représenté par la zone montante du tronçon B (soit huit canaux en série) en fonction du débit d'eau à $22^{\circ} \mathrm{C}$.

Compte tenu du manque d'informations théoriques sur les phénomènes hydrodynamiques dans les échangeurs à plaques, nous avons appliqué à nos résultats les formules classiquement utilisées pour les conduits tubulaires lisses de section quelconque.

La loi générale établissant la valeur de la perte de charge $(\Delta \mathrm{P})$ en fonction des paramètres caractéristiques des conditions d'écoulement $[12,13]$ s'écrit :

$$
\Delta \mathrm{P}=\frac{2 . f .1}{\mathrm{D}} \rho \cdot \mathrm{v}^{2} \quad\{1\}
$$

1 est la longueur du circuit considéré ;

D est le diamètre hydraulique du circuit, dans le cas d'un échangeur à plaques il correspond à l'expression

$4 \times$ section droite

périmètre mouillé

$\rho$ est la masse volumique du fluide à sa température moyenne ;

v est la vitesse linéaire du fluide ;

f est le facteur de friction dont la valeur est directement fonction du nombre de Reynolds. Pour des zones déterminées de turbulence, $\mathrm{f}$ peut être traduit par la relation :

$$
\mathrm{f}=\mathrm{a} \cdot \mathrm{Re}^{\mathrm{m}}
$$

a et $m$ sont alors des constantes dépendant de la rugosité du matériau, et des caractéristiques géométriques des circuits. 
Les circuits hydrauliques des échangeurs à plaques étant constitués de canaux identiques, diversement combinés, la relation $\{1\}$ peut s'écrire :

$$
\Delta \mathrm{P}=\mathrm{k} \cdot \mathrm{Re}^{\mathrm{m}} \cdot \rho \cdot \mathrm{v}^{2} \cdot \mathrm{n} \quad\{2\}
$$

$\mathrm{k} \quad$ est une constante : $\mathrm{k}=\frac{2 \mathrm{aL}}{\mathrm{D}}$;

L est la longueur d'un canal ;

n est le nombre de passes du circuit.

A partir des valeurs expérimentales de pressions et débits obte$\Delta \mathrm{P}$

nues, en traçant $L_{n}\left(\frac{}{n \cdot \rho . v^{2}}\right)$ en fonction de $L_{n} \operatorname{Re}$ (fig. 3), nous obtenons une droite de pente $\mathrm{m}$ et d'ordonnée à l'origine $\mathrm{L}_{\mathrm{n}} \mathrm{k}$. La valeur de $\mathrm{m}$ égale $-0,10$.

Dans le cas des tubes cylindriques, cela signifierait que l'écoulement s'effectue en régime de turbulence moyenne. En effet, il est

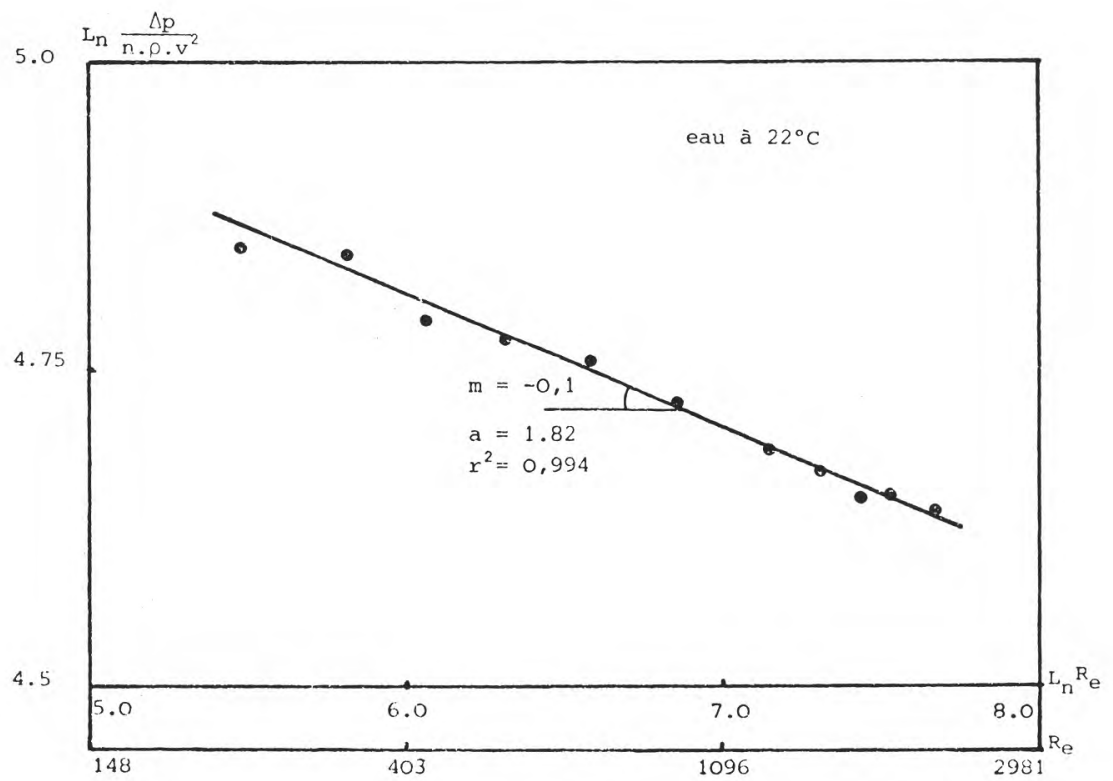

fig. 3

Identification sur un canal de l'échangeur du modèle de perte de charge appliqué aux circuits tubulaires. 
admis que la valeur de $\mathrm{m}$ peut atteindre $-0,25$ en régime faiblement turbulent, qu'elle diminue lorsque la turbulence augmente, et qu'elle tend vers zéro à turbulence élevée $\left(\operatorname{Re}>10^{+5}\right)$ [14]. Dans ce cas seulement, la perte de charge varie avec le carré de la vitesse linéaire.

Par contre, dans nos conditions expérimentales $(150<\operatorname{Re}<3000)$, la perte de charge varie en fonction de la vitesse à la puissance 1,90 . Le régime d'écoulement est néanmoins turbulent car la valeur du nombre de Reynolds n'a plus la même signification dans les canaux rectangulaires et ondulés constitués par l'empilement des plaques. L'identification de la relation $\{2\}$ conduit simplement à une valeur de a $(1,82)$ très supérieure à celles correspondant aux circuits cylindriques [13]. Mais, globalement le modèle reste applicable.

\section{Evolution des performances hydrodynamiques et thermiques du pasteurisateur}

\subsection{Généralités}

Les performances d'un échangeur de chaleur sont généralement caractérisées par le coefficient général d'échange, noté U. Il traduit le flux de chaleur échangé $(\Phi)$ par unité de surface (A) lorsque la différence de température moyenne logarithmique (représentée dans le cas d'une circulation méthodique par la moyenne logarithmique des écarts de températures extrêmes, $\left.\Delta \theta_{\mathrm{m} 1}\right)$ est de $1^{\circ} \mathrm{C}$ :

$$
\mathrm{U}=\frac{\Phi}{\mathrm{A} \cdot \Delta \theta_{\mathrm{m} 1}} \quad\{3\}
$$

Il s'exprime en kilocalories par heure, mètre carré et degré centigrade. En fait, sa valeur absolue n'a de signification qu'accompagnée du programme thermique assuré par le pasteurisateur, donc des valeurs de débits et températures des fluides.

Son inverse $(1 / \mathrm{U})$ est la somme des résistances thermiques s'opposant au transfert de chaleur c'est-à-dire celle de la paroi $\left(\frac{\mathrm{e}}{\lambda}\right)$ et celles des couches limites créées par les deux fluides $\left(\frac{1}{\mathrm{~h} 1}\right.$ et $\left.\frac{1}{\mathrm{~h} 2}\right)$.

$$
\frac{1}{\mathrm{U}}=\frac{1}{\mathrm{~h} 1}+\frac{\mathrm{e}}{\lambda}+\frac{1}{\mathrm{~h} 2}
$$

Le calcul des coefficients de film (h1 et h2) s'effectue au moyen des invariants de similitude utilisés pour les transferts de chaleur entre un fluide en mouvement turbulent et la paroi d'un cylindre lisse.

Ces invariants sont les nombres de Reynolds $\left(\mathrm{R}_{\mathrm{e}}\right)$, de Prandtl $\left(\mathrm{P}_{\mathrm{r}}\right)$ et de Nusselt $\left(\mathrm{N}_{\mathrm{u}}\right)$. Ils sont reliés par une formule de type :

$$
\mathrm{N}_{\mathrm{u}}=\mathrm{a} \cdot \mathrm{R}_{\mathrm{e}}^{\mathrm{b}} \cdot \mathrm{P}_{\mathrm{r}} \mathrm{c} \quad\{5\}
$$




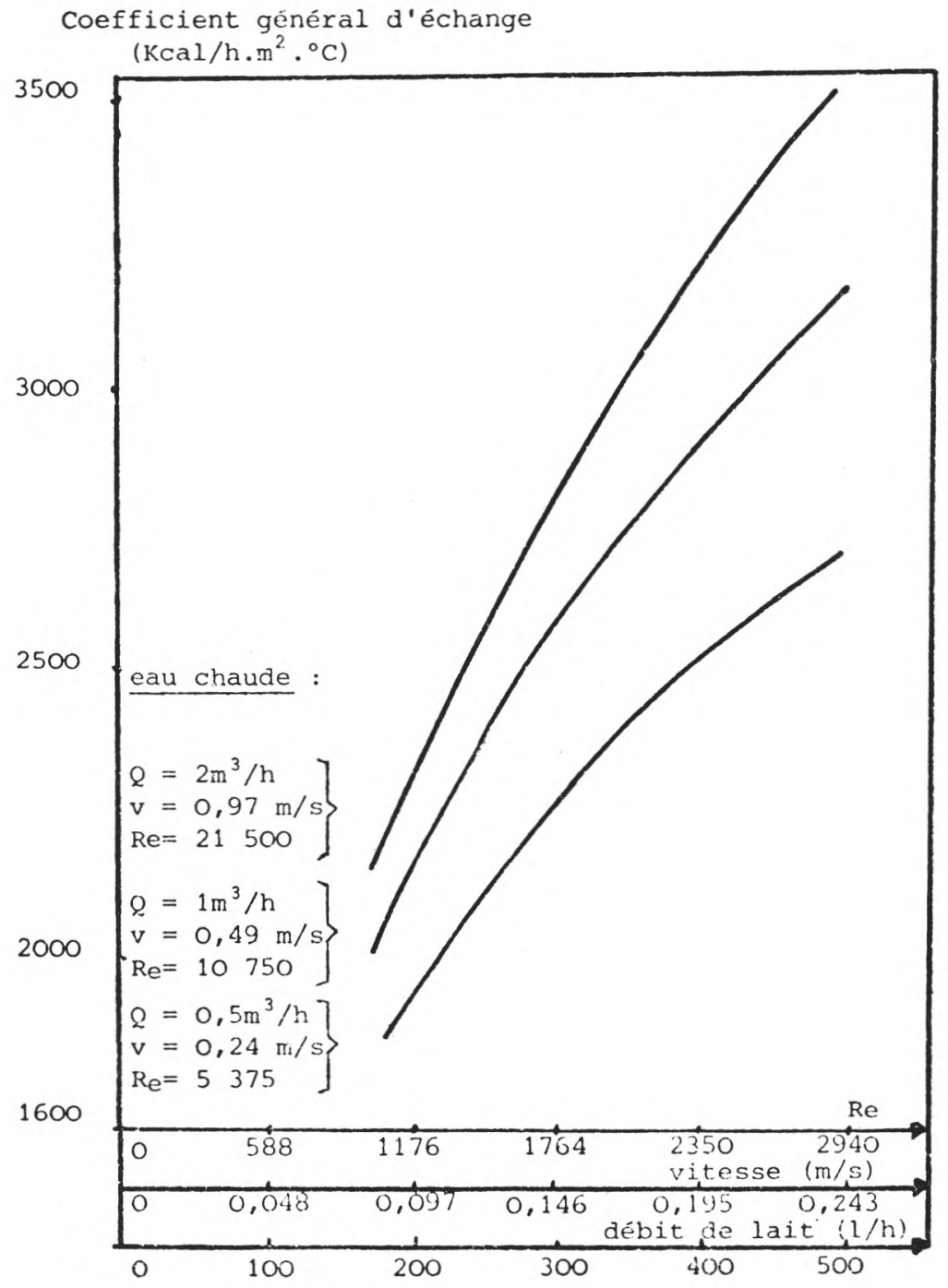

fig. 4

Variation du coefficient global d'échange de la zone de chauffage en fonction des caractéristiques d'écoulement du lait pour trois débits d'eau chaude (courbes simulées à partir du modèle hydrodynamique représenté par les équations $\{4\}$ et $\{5\}$ ). 
Les valeurs des coefficients numériques ( $a, b$ et $c$ ), dans une plage de turbulence établie, sont fonction de la géométrie de l'échangeur considéré. Dans le cas du pasteurisateur V7 nous avons utilisé celles fournies par Vicarb.

Au fur et à mesure de la pasteurisation du lait, un dépôt se fixe sur les plaques. S'il modifie les conditions d'écoulement du lait, il se traduit surtout par une augmentation progressive de la résistance thermique s'opposant au transfert de chaleur. La valeur du coefficient général d'échange décroît et la température de pasteurisation ne peut être maintenue qu'en augmentant d'autant la valeur de la différence de température moyenne logarithmique (fig. 8.).

Il est facile de concevoir que les performances hydrodynamiques et thermiques du pasteurisateur seront donc influencées par son degré d'encrassement.

\subsection{Encrassement nul}

La période d'encrassement nul, de courte durée, correspond aux premières minutes de la pasteurisation.

Afin de caractériser, lors de cette période, les performances du pasteurisateur, nous présentons figures 4,5 et 6 l'évolution du coefficient général d'échange, simulé à partir des équations $\{4\}$ et $\{5\}$, en fonction des débits des fluides dans les zones de chauffage, de récupération et de réfrigération.

Il apparaît, figure 4 , que le coefficient général d'échange de la zone de chauffage, est plus sensible à l'augmentation du débit de lait qu'à celle du débit d'eau chaude. L'explication réside à la figure 7 dans la mesure où la résistance thermique (inverse du coefficient local d'échange) de la couche limite est plus élevée côté lait que côté eau chaude. La même figure permet de constater que les conditions d'expérience sont, vis-à-vis des coefficients de transfert, nettement plus défavorables pour le lait et l'eau glycolée que pour l'eau chaude. Cette situation se traduit (fig. 5 et 6) par des coefficients généraux d'échange plus faibles que ceux de la zone de chauffage (fig. 4). Mais les débits (ou vitesses linéaires d'écoulement) ne sont pas les seuls paramètres influençant la valeur des coefficients d'échange. La figure 7 permet de mesurer l'importance de la température moyenne du lait zone par zone : de $13^{\circ} \mathrm{C}$ à $77^{\circ} \mathrm{C}$, le coefficient local d'échange est amélioré d'environ 36 p. 100 quel que soit le débit de pasteurisation. Comme il est souhaitable que ce dernier reste constant, aucune amélioration des coefficients d'échange n'est possible dans les "zones froides " du pasteurisateur.

\subsection{Observation et effet de l'encrassement}

L'examen visuel de l'encrassement apporte plusieurs enseignements : 


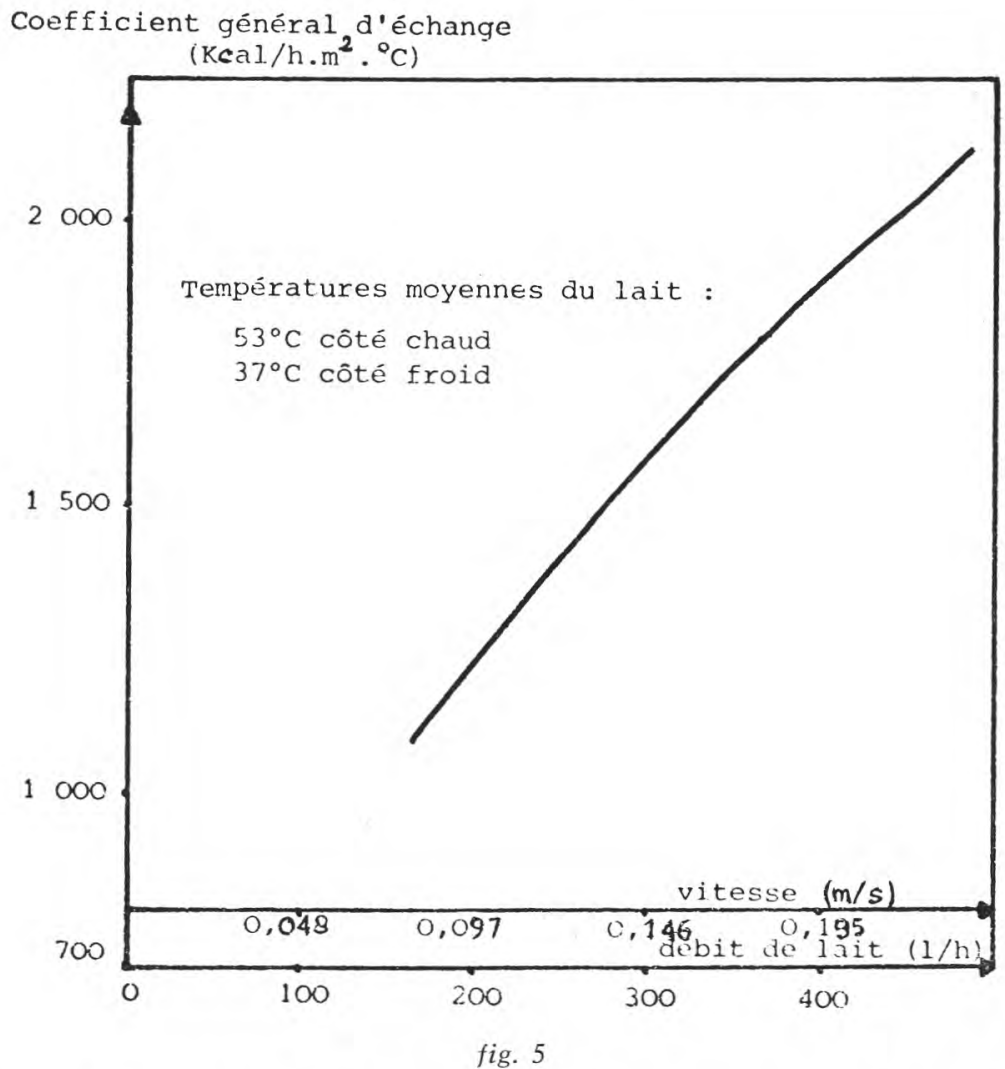

Variation du coefficient général d'échange de la zone de récupération de chaleur en fonction du débit de lait (courbes simulées à partir du modèle hydrodynamique représenté par les équations $\{4\}$ et $\{5\}$ ).

- seules les plaques en contact avec le lait chauffé à plus de $65^{\circ} \mathrm{C}$ présentent des dépôts solides. Les autres plaques, après rinçage à l'eau froide, ne comportent pas de traces visibles de lait ;

- les dépôts sont de couleur blanche. Il est logique de penser qu'ils sont du même type (50 à 60 p. 100 de protéines, 25 à 35 p. 100 de matières minérales) que ceux analysés par Lyster [15] et Burton [16]. Nous n'avons jamais observé une quelconque caramélisation du dépôt même lorsque, accidentellement, la circulation de lait a pu être interrompue pendant quelques minutes;

- les dépôts ont un aspect spongieux. De nombreux vides ou alvéoles, de taille variable, expliquent, au moins partiellement, cela. Leur origine semble être la rétention mécanique de gaz, insolubilisés par l'élévation de température. Burton [16] pense que ces gaz, en 


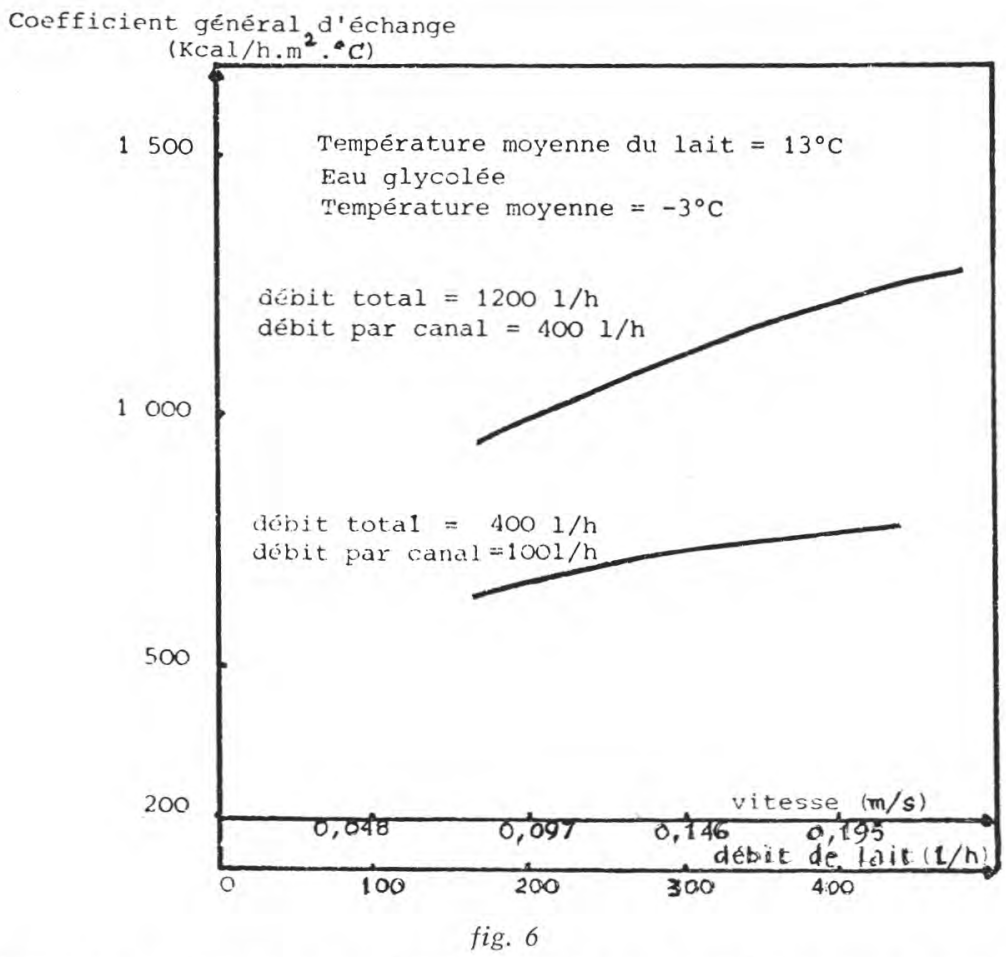

Evolution du coefficient général d'échange de la zone de réfrigération en fonction du débit de lait pour deux débits d'eau glycolée (courbes simulées à partir du modèle hydrodynamique représenté par les équations $\{4\}$ et $\{5\})$.

contact avec la paroi chaude, favorisent la formation de dépôts. Il préconise de travailler à une pression suffisante pour éviter leur insolubilisation ;

- la répartition des dépôts est homogène au niveau de chaque plaque. Les conditions d'écoulement expérimentées (vitesses linéaires comprises entre 0,1 et $0,2 \mathrm{~m}$ par seconde) évitent donc la formation de zones mortes. Ce n'est plus le cas pour des vitesses d'écoulement de l'ordre de $0,05 \mathrm{~m}$ par seconde ;

- le degré d'encrassement du pasteurisateur varie selon les essais en fonction de la qualité des laits traités. La quantification de ce phénomène est délicate ;

- le degré d'encrassement des plaques varie selon leur position dans le pasteurisateur. Par exemple, dans la zone de chauffage, l'encrassement augmente avec la température du lait. Ceci est confirmé par l'évaluation quantitative des dépôts par pesée : la première passe ne fixe que 20 à 35 p. 100 des dépôts totaux de la zone de chauffage, 
le reste étant fixé au niveau de la deuxième passe. En règle générale, dans un pasteurisateur, les canaux les plus encrassés seront ceux où le lait atteint les températures les plus élevées.

Par opposition dans la zone de chambrage où la température du lait peut être considérée comme constante, nous avons constaté que la quantité de dépôts diminue en fonction du temps de séjour. 20 à 25 p. 100 des dépôts totaux sont fixés à la première plaque, seulement 10 à 15 p. 100 à la dernière.

Sur le plan thermique, le dépôt crée une résistance supplémentaire au transfert de chaleur. Le tableau 1 permet de comparer les valeurs relatives de cette résistance, selon le degré d'encrassement, et celles que constituent la paroi et les couches limites. Les résistances thermiques d'encrassement que nous indiquons correspondent aux valeurs finales mesurées lors des essais [17]. Pour les faibles encrasse-

\section{TABLEAU 1}

Résistances thermiques s'opposant au transfert de chaleur dans la zone de chauffage

(toutes les résistances sont exprimées en $\mathrm{h} . \mathrm{m}^{2}{ }^{\circ} \mathrm{C} / \mathrm{Kal}$ et correspondent au programme thermique présenté au paragraphe II)

Echangeur non encrassé

\begin{tabular}{c|c|c|c}
\hline $\begin{array}{c}\text { Résistance } \\
\text { de la } \\
\text { paroi } \\
\text { métallique }\end{array}$ & $\begin{array}{c}\text { Résistance de la } \\
\text { couche limite } \\
\text { côté lait } \\
\text { débit }: 3001 / \mathrm{h}\end{array}$ & $\begin{array}{c}\text { Résistance de la } \\
\text { couche limite } \\
\text { côté eau chaude } \\
\text { débit }: 900 \mathrm{l} / \mathrm{h}\end{array}$ & $\begin{array}{c}\text { Résistance } \\
\text { totale }\end{array}$ \\
\hline $0,5.10^{-4}$ & $2,5.10^{-4}$ & $1.10^{-4}$ & $4.10^{-4}$ \\
\hline
\end{tabular}

Echangeur encrassé

\begin{tabular}{c|c|c|c}
\hline $\begin{array}{c}\text { Degré } \\
\text { d'encrassement }\end{array}$ & $\begin{array}{c}\text { Résistance de } \\
\text { l'échangeur }\end{array}$ & $\begin{array}{c}\text { Résistance du } \\
\text { dépôt }\end{array}$ & $\begin{array}{c}\text { Résistance } \\
\text { totale }\end{array}$ \\
\hline nul & $4.10^{-4}$ & néant & $4.10^{-4}$ \\
faible & $4.10^{-4}$ & $3.10^{-4}$ & $7.10^{-4}$ \\
moyen & $4.10^{-4}$ & $9.10^{-4}$ & $13.10^{-4}$ \\
fort & $4.10^{-4}$ & $16.10^{-4}$ & $20.10^{-4}$ \\
\hline
\end{tabular}




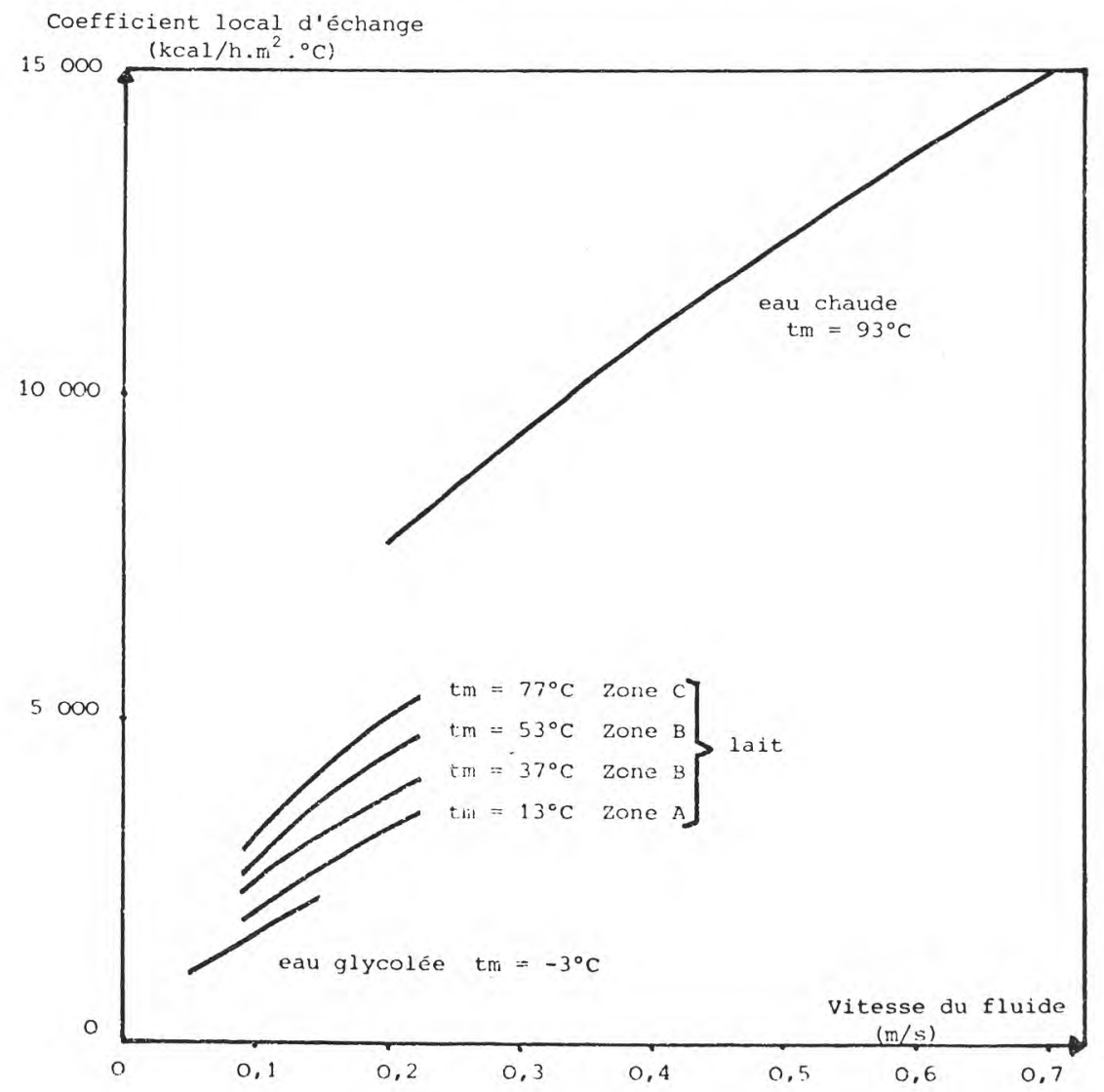

fig. 7

Evolution des coefficients locaux d'échange dans les conditions d'expérience pour chacun des fluides (courbes simulées à partir du modèle hydrodynamique représenté par les équations $\{4\}$ et $\{5\}$ ).

ments, la résistance thermique constituée par le dépôt, est légèrement inférieure à la somme des autres résistances. Par contre, elle lui est quatre fois supérieure aux forts encrassements. Les conditions de pasteurisation étant relativement proches, ceci montre combien la qualité du lait traité peut influencer l'encrassement et son évolution [17].

En règle générale, les problèmes d'encrassement n'ont pas reçu de solutions satisfaisantes. Ils conditionnent pourtant le fonctionnement des unités industrielles (sans se limiter à la pasteurisation) et le dimensionnement des matériels. Par exemple, la valeur de résistance thermique attribuée à l'encrassement lors du calcul d'un pasteu- 


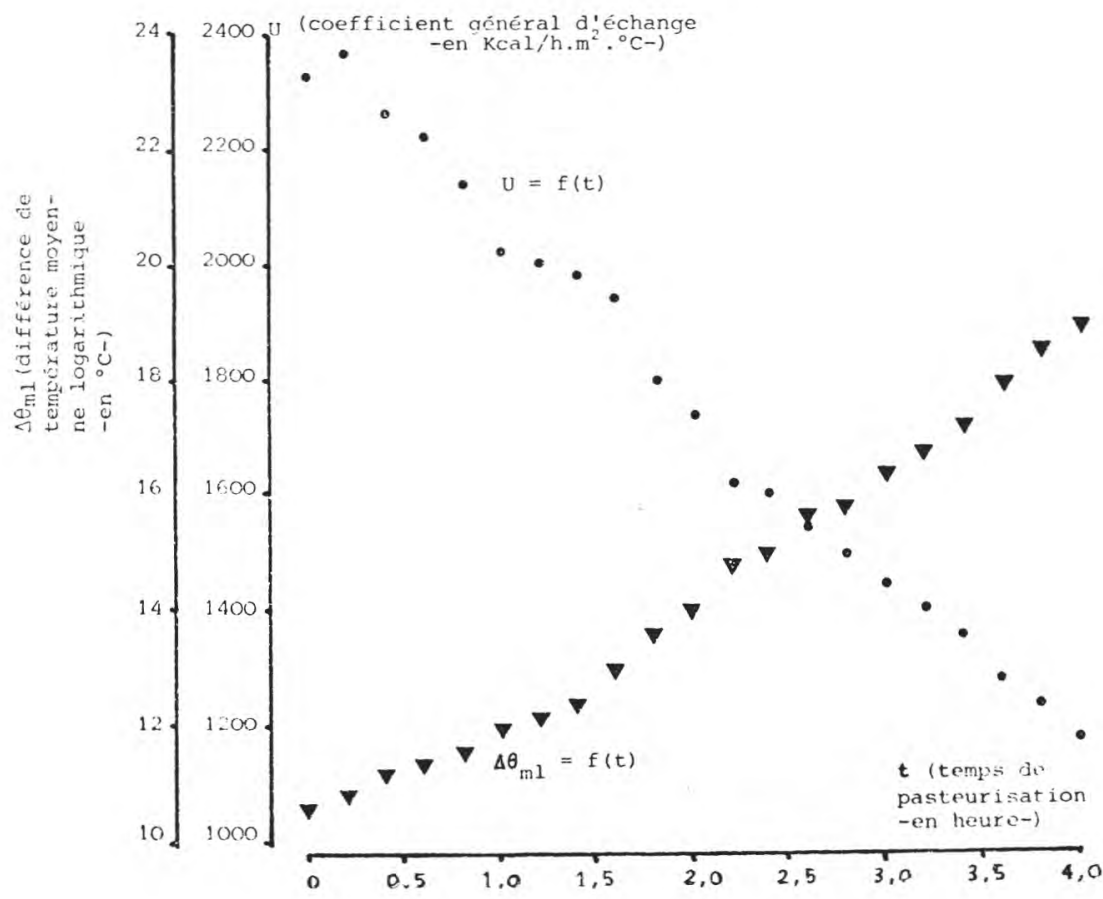

fig. 8

Evolution, du fait de l'encrassement, du coefficient général d'échange et de la différence de température moyenne logarithmique en fonction du temps de pasteurisation.

risateur va influencer sa taille (surface d'échange) et par la suite sa durée de fonctionnement entre deux nettoyages successifs.

La mesure en ligne du degré d'encrassement, la définition de seuils économiques à ne pas dépasser afin de faciliter le nettoyage ou de réduire les dépenses énergétiques, le choix de matériaux limitant ou évitant la formation des dépôts pourraient être à la base de progrès minimisant l'importance de ce problème.

\subsection{Régulation de la température de pasteurisation}

La formation puis l'accroissement du dépôt au fur et à mesure de la pasteurisation se traduisent par la diminution progressive du coefficient général d'échange (U), du fait d'une résistance thermique supplémentaire. Le maintien de la température de pasteurisation du lait, impose de compenser cette diminution en conservant la quantité de chaleur transférée $(\Phi)$. Cela est réalisé en augmentant la différence de température moyenne logarithmique $\left(\Delta \theta_{\mathrm{m} 1}\right)$. Un exemple de ce double phénomène est présenté à la figure 8 : au cours d'une 
pasteurisation de $4 \mathrm{~h}$, le coefficient général d'échange décroît d'environ 50 p. 100 alors que la différence de température moyenne logarithmique passe de $10,6^{\circ} \mathrm{C}$ à $19,2^{\circ} \mathrm{C}$.

En pratique, une boucle de régulation asservit à la température de pasteurisation du lait soit la température soit le débit du fluide de chauffage. Nous avons choisi cette dernière solution. La température de pasteurisation du lait a ainsi pu être maintenue dans une plage de $\pm 0,5^{\circ} \mathrm{C}$ par rapport à la température de consigne.

Les choix de l'actionneur et du régulateur électronique constituant la boucle de régulation sont délicats, en particulier à l'échelle industrielle, du fait des performances exigées et de l'effet de l'encrassement.

\section{Opérations de nettoyage}

Le terme nettoyage désigne l'ensemble des opérations de désinfection, rinçage et nettoyage associées à la pasteurisation du lait [18]. En règle générale, la désinfection précède la pasteurisation proprement dite. Lorsqu'elle fait appel à un procédé chimique, elle est suivie par un rinçage avec de l'eau bactériologiquement propre qui précède l'introduction du lait. Après pasteurisation, le rinçage à l'eau, entraînant le lait résiduel, est suivi par le nettoyage alcalin qui élimine la majeure partie des dépôts solides fixés aux plaques, en particulier leurs constituants organiques. Après rinçage à l'eau, un éventuel lavage acide élimine les dépôts solides de type minéral. Il est suivi par le rinçage final.

Nous avons appliqué ce protocole en utilisant une solution d'hypochlorite de sodium à $150 \mathrm{mg}$ de chlore actif pour la désinfection, une solution de soude à $20 \mathrm{~g}$ par litre pour le nettoyage alcalin et une solution d'acide nitrique à $10 \mathrm{~g}$ par litre pour le nettoyage acide.

Toutes les opérations ont été réalisées automatiquement, sous la conduite du minicalculateur, avec des temporisations identiques à celles adoptées sur les installations de nettoyage en place (N.E.P.) couramment utilisées dans l'industrie laitière. Les débits et températures choisis répondaient aux mêmes impératifs.

L'examen visuel des plaques a montré la nécessité du lavage acide afin d'éliminer l'important dépôt minéral résultant du lavage alcalin. Néanmoins, l'effet négatif de ce dépôt, en particulier sur les conditions d'encrassement, devrait être étudié. En effet, Lund et Bixby [19] ont montré qu'un prédépôt de phosphate de calcium provoque certes une diminution du coefficient général d'échange de l'ordre de 50 p. 100 , mais évite tout encrassement lors de la pasteurisation qui suit. Ce résultat établi à partir d'expériences limitées à $3 \mathrm{~h}$ devrait être confirmé avant d'envisager une quelconque application.

L'efficacité des deux lavages est démontrée par le parfait état des plaques et des tubulures de liaison à la fin des opérations. 
L'efficacité de la désinfection est démontrée par la qualité des laits pasteurisés qui n'ont jamais subi, même après 24 h de stockage, de recontaminations notables.

En conclusion, les conditions de désinfection, rinçage et nettoyage habituelles dans l'industrie laitière, s'appliquent sans problème au matériel testé, et satisfont à son fonctionnement.

Néanmoins, il apparaît que bien des progrès restent à faire pour gérer de façon plus économique l'ensemble des opérations de nettoyage [18]. Le développement de capteurs spécifiques propres au contrôle des différentes phases, leur modélisation au moyen de paramètres hydrodynamiques [10] doivent permettre de réduire les coûts principaux (immobilisation des matériels, main-d'œuvre, produits chimiques, épuration des rejets).

\section{Qualité bactériologique des laits traités}

Alais [20] précise les principales caractéristiques bactériologiques imposées par la législation française aux laits pasteurisés.

Par opposition à la stérilisation qui vise en outre la destruction des spores bactériennes, la pasteurisation ne provoque qu'une diminution du nombre des micro-organismes présents sous forme végétative, la réduction étant particulièrement forte pour ceux d'entre eux qui sont pathogènes. En conséquence, la qualité bactériologique du lait pasteurisé sera, pour un traitement donné, directement liée à celle du lait cru.

Le comptage des germes totaux contenus dans le lait (selon les méthodes classiques de culture sur milieu gélosé en boîte de pétri après dilution) avant et après la pasteurisation, permet de vérifier son efficacité. Le comptage des coliformes sur un milieu gélosé à base de lactose-désoxycholate met en évidence une éventuelle recontamination du lait au sein de l'échangeur (en cas de défaut d'étanchéité ; par exemple entre les circuits de préchauffage et de refroidissement).

L'interprétation de ces différents comptages doit rester prudente; un lait pasteurisé peut contenir trop de germes, malgré une pasteurisation correcte, s'il est recontaminé au contact de parois non stériles. Indépendamment de la pasteurisation, les modalités de nettoyage et de désinfection de l'installation ont donc une importance considérable.

En pratique, le comptage des coliformes a toujours donné un résultat négatif. Aucun coliforme ne survit à la pasteurisation réalisée, selon les essais, entre 80 et $85^{\circ} \mathrm{C}$ à des débits et avec des temps de chambrage respectivement compris entre 220 et 4101 par heure et entre 16,4 et 8,8 , s.

L'échangeur ne présente donc aucun défaut conduisant à une recontamination du lait traité ; les opérations de lavage et de désinfection ont un effet satisfaisant.

Par ailleurs, avant traitement, les laits (crus, entiers et de grand mélange) comptaient un nombre total de germes compris entre $1,8.10^{+6}$ 
et $10^{+7}$ selon les livraisons. Après pasteurisation, ils comptaient un nombre total de germes de l'ordre de $10^{+3}$. Ce résultat est conforme aux normes imposées aux laits pasteurisés mais traduit la présence d'une flore thermorésistante importante.

En conclusion, le fonctionnement sur le plan bactériologique du pasteurisateur est satisfaisant.

\section{CONCLUSIONS}

Aucun problème technique ne semble empêcher l'utilisation pour la pasteurisation du lait d'un échangeur à plaques Vicarb. Les essais sont positifs pour ce qui concerne le modèle V7. Leur extrapolation aux modèles de taille supérieure est sans problème pour la plupart des paramètres étudiés, bien qu'une certaine prudence s'impose pour ce qui concerne les conditions d'encrassement et de nettoyage.

Cette conclusion s'étend à tous les traitements thermiques de l'industrie laitière et fromagère, plus simples que la pasteurisation (réfrigération, refroidissement, thermisation) ainsi qu'à tous les produits dérivés du lait excepté, peut-être la crème pour laquelle des essais particuliers devraient être effectués.

Par ailleurs, le traitement U.H.T. (ultra haute température) pourrait être envisagé avec les échangeurs Vicarb sous réserve d'essais conduisant à la mise au point d'un procédé original.

En nous plaçant dans l'optique de l'utilisation de ce matériel dans les industries agro-alimentaires en général, nous arrivons également à une conclusion positive, dans la mesure où le problème qui nous sert de référence est l'un des plus délicat qui soit. Seuls quelques produits très particuliers nécessiteraient du fait de leur composition ou de leur viscosité des essais préalables (œufs, sirops, etc.).

Sur le plan scientifique, ce travail a, par contre, révélé les limites des connaissances actuelles et autant d'axes de recherches possibles.

Les lois théoriques, décrivant le fonctionnement des échangeurs à plaques, sont une simple adaptation de celles relatives aux échangeurs tubulaires. En conséquence, l'influence de la géométrie des plaques n'est pas quantifiée.

Les mécanismes et les cinétiques d'encrassement restent à étudier afin d'améliorer les conditions de travail actuelles et la conception des matériels. Le développement de capteurs spécifiques (mesure du degré d'encrassement, de nettoyage, de rinçage), le choix de matériaux moins coûteux et/ou plus performants que les aciers inoxydables actuellement utilisés peuvent également être source de progrès.

Reçu pour publication en novembre 1978.

\section{Remerciements}

Nous remercions la Société Vicarb et le Ministère de l'Industrie dont l'aide financière a permis la mise en cuvre de la plate-forme expérimentale et la conduite des essais. 


\section{Références bibliographiques}

[1] Vinconneau (M.) et Crevel (M.) (1976). - Les échangeurs à plaques et à spirales dans l'industrie sucrière. Ind. Alim. Agri., 7-8, 825.

[2] Kern (D. Q.) and Kraus (A. D.) (1972), - Extended surface heat transfert. Edit. Mac Graw-Hill.

[3] Handbook of heat transfert. Edit. Rohsenow W. and Hartnett J. - Mac Graw-Hill (1973).

[4] Techniques de l'ingénieur (J3). Transfert de chaleur. Transfert de matière.

[5] Kessler (H. G.) und Walenta (W.) (1977), - Prüfung und technische Begutachtung des Platten. Kurzzeiterhitzers und des Platten. Hocherhitzers " Ahlborn Waritherm 40 ». Deutsche Milchwirtschaft, 43, 1532.

[6] Ministère de L'Agriculture (1978). - Enquête annuelle d'entreprises 1974. I.A.A., étude $\mathrm{n}^{\circ} 163$, juin.

[7] Daufin (G.), Kerherve (F. L.) et Richard (J.) (1977). - Influence de l'état de surface de l'acier inoxydable sur son aptitude au nettoyage et sur sa sensibilité à la corrosion par piqûres. Ind. Alim. Agr., 94, 1, 43.

[8] Daufin (G.), Desestret (A.), Kerherve (F. L.), Michel (F.), Tremoureux (Y.) et VAllier (G.) (1977), - Etude de la corrosion d'aciers inoxydables ferritiques et austénitiques et d'alliages à hautes caractéristiques mécaniques dans les conditions de l'industrie laitière. Compte rendu de fin d'étude D.G.R.S.T. (action métallurgie).

[9] RaO (M.) and LoNCIN (M.) (1974). - Residence time distribution and its role in continuous pasteurization. Lebensm. Wiss. u. Technol., 7, 5.

[10] Thor (W.) und Loncin (M.) (1978). - Reinigen, Desinfizieren und Nachspülen in der Lebensmittel. Industrie Chem. Ing. Tech., 50, 3, 188.

[11] Levenspiel (O.) and Bischoff (K. B.) (1963). - Patterns of flow in chemical process vessels, Advances in chemical engineering. Edit. Drew T, Hoopes J. and Vermeulen T. Academic Press, 4, 94.

[12] Perry (R. H.) and Chilton (C. H.) (1973). - Fluid and particle mechanics, 5-21. Chemical Engineers'Handbook (4). Edit. Mac Graw-Hill.

[13] Loncin (M.) (1976). - Génie Industriel Alimentaire. Aspects fondamentaux. Edit. Masson, p. 101.

[14] Leniger (H. A.) and Beverloo (W. A.) (1975). - Food Process Engineering. Edit. Reidel D., p. 26.

[15] LysteR (R. L. J.) (1965). - The composition of milk deposits in an ultra high temperature plant. J. Dairy Res., 32, 203.

[16] Burton (H). (1968). - Reviews of the progress of Dairy Science. J. Dairy Res., 35, 317.

[17] Lalande (M.) et Corrieu (G.). - Evaluation du degré d'encrassement d'un échangeur de chaleur à plaques (en cours de publication).

[18] Daufin (G.) (1977). - Procédés modernes de nettoyage et de désinfection dans l'industrie laitière. Ind. Alim. Agri., 94, 148.

[19] Lund (D. B.) and BIXBY (D.) (1975). - Fouling of heat exchange surface by milk. Proc. Biochem., 10, 52.

[20] Alais (C.) (1975). - Science du lait (principales techniques). Edit. SEP, $3^{\mathrm{e}}$ édition, p. 524. 